\title{
Inhibición Conductual y su Relación con los Trastornos de Ansiedad Infantil
}

\author{
Behavioral Inhibition and Relationship with Childhood Anxiety Disorders
}

\author{
Alfonso Ordóñez-Ortega \\ Lourdes Espinosa-Fernández \\ Luis-Joaquín García-López \\ José-Antonio Muela-Martínez \\ Universidad de Jaén, España
}

(Rec: 1 de agosto de 2012 / Acept: 24 de junio de 2013)

\begin{abstract}
Resumen
La literatura científica ha mostrado que la inhibición conductual (IC) se caracteriza por la presencia de ansiedad ante estímulos desconocidos, lo que conlleva conductas de retraimiento y evitación social. El presente estudio realiza una revisión bibliográfica sobre la IC y su relación con los trastornos de ansiedad en la infancia. Dado que la IC puede observarse en edades muy tempranas, la literatura ha examinado su papel predictor en el desarrollo de trastornos de ansiedad. Pese a ello, los estudios revisados apuntan a que no todos los niños ansiosos muestran un comportamiento inhibido ni todos los niños con IC sufren trastornos de ansiedad. Dada esta disparidad, sería necesario estudiar qué otros factores están implicados. Asimismo, los datos apuntan a que una detección precoz de la IC permitiría desarrollar programas de prevención de los trastornos de ansiedad en la infancia.

Palabras clave: ansiedad, inhibición conductual, infancia
\end{abstract}

\begin{abstract}
The literature has revealed that Behavioral inhibition (BI) is characterized by the presence of anxiety to unknown stimuli, which leads to withdrawal behaviors and social avoidance. The present study is aimed at revising the state-of-the-art on $\mathrm{BI}$ and examining its role that plays of childhood anxiety disorders. As the IB can be observed at very early ages, the literature has examined its predictive role in the development of anxiety disorders. Despite this, the reviewed studies suggest that not all anxious children show comorbid BI nor all inhibited children have anxiety disorders. Given this disparity, it would be necessary to consider what other factors are involved in the anxiety disorders. Furthermore, the data suggest that early detection of the BI would contribute to develop prevention programs for anxiety disorders in childhood.

Key words: anxiety, behavioral inhibition, childhood
\end{abstract}

\section{Introducción}

La inhibición conductual (IC) es una variable temperamental que genera timidez, retraimiento, evitación social, inquietud o malestar social, además de miedo ante personas, objetos y/o situaciones desconocidas (García-Coll, Kagan y Reznick, 1984). La variable IC ha sido objeto de estudio en considerables investigaciones durante las últimas décadas. En concreto, desde el año 2000, un gran número de estudios se han centrado en conocer la relación existente entre la IC y la psicopatología, las variables asociadas con la inhibición y los métodos para evaluarla. Y también, aunque en menor medida, se ha investigado su delimitación conceptual, el papel de la cultura en la IC, la evaluación de la competencia social y la relación con los correlatos neurobiológicos (Guerra, Martín y Arnaiz, 2011).

Este artículo realiza una revisión de las investigaciones llevadas a cabo sobre la IC con el objetivo de establecer

Correspondencia: Alfonso Ordóñe-Ortega. Departamento de Psicología. Campus Las Lagunillas. Edificio Humanidades y Ciencias de la Educación II (C5). Universidad de Jaén, 23071. Jaén (España). Tel.: (+34) 953 211814. Fax: (+34) 953211881.

Correo electrónico: aordonez.ujaen@gmail.com 
una visión sobre su estado actual. Para ello, se centra principalmente en las características más importantes que la define e investiga la relación que la inhibición tiene con el desarrollo de trastornos de ansiedad en los niños. Además, estudia la relación de la inhibición con otras variables que puedan intervenir en el desarrollo de patologías ansiosas, expone los procedimientos de evaluación utilizados para realizar una detención precoz de la IC y, por último, adelanta las posibles líneas de estudio futuras.

\section{Características de la variable IC}

La IC es el rasgo temperamental definido por el miedo extremo ante la novedad (Kagan, Reznick y Snidman, 1987). $\mathrm{Al}$ igual que otras variables temperamentales, la IC muestra una disposición básica, es relativamente consistente, está determinada biológicamente y es sensible a la influencia de variables contextuales (Goldsmith et al., 1987). Los niños inhibidos experimentan una gran ansiedad ante estímulos desconocidos, inciertos o cambiantes, por lo que reaccionan inicialmente mostrando signos de sufrimiento, moderación del comportamiento y latencias largas para interactuar. También, suelen dejar de jugar, hablar y tratan de permanecer cerca de la seguridad que ofrecen sus padres (Kagan, Snidman y Arcus, 1998). De hecho, algunos autores han argumentado que la IC es la manifestación observable del rasgo de personalidad de la neurosis (Craske, 1997) o tendencia persistente a experimentar estados emocionales negativos (Matthews, Deary y Whiteman, 2003). Asimismo, se relaciona con la baja extraversión, otro rasgo de personalidad asociado con una menor participación en actividades sociales y con individuos menos reivindicativos que prefieren observar las situaciones antes de participar en ellas (Matthews et al., 2003). En esta línea, Vreeke y Muris (2012) exponen que los padres de niños clasificados como clínicamente ansiosos, informan que sus hijos presentan niveles más elevados de neuroticismo e IC, así como niveles inferiores de extroversión en comparación con padres de niños no ansiosos. Además, los niveles elevados de IC y neuroticismo fueron predictores de presentar un trastorno de ansiedad en el futuro.

Aunque la presencia de IC se observa muy tempranamente en la vida (Kagan, Reznick, Snidman, Gibbons y Johnson, 1988), para su detección, es necesario considerar el período evolutivo en el que se encuentra el niño. En este sentido, la IC no debe confundirse con la reacción normal de ansiedad al extraño en la que los niños de 7-8 meses muestran respuestas ansiosas ante personas desconocidas cuando ya distinguen la figura de vínculo, generalmente la madre, de los extraños (Spitz, 1961). Spitz señala que este tipo de ansiedad expresa un desarrollo afectivo y madurativo adecuado, a diferencia de la conducta inadaptada que la IC puede mantener durante toda la infancia, adolescencia (Pfeifer, Goldsmith, Davidson y Rickman, 2002) e incluso en la adultez (Kagan, Snidman, Kahn y Towsley, 2007). En función del sexo, las niñas tienden a mostrar niveles de IC más altos y estables que los niños (Essex, Klein, Slattery, Goldsmith y Kalin, 2010; Kerr, Lambert, Stattin y Klackenberg-Larsson, 1994); estas diferencias son atribuidas al efecto de los estereotipos del rol sexual, ya que la inhibición resulta socialmente más aceptable en el sexo femenino que en el masculino (Kerr et al., 1994).

En relación con los contextos donde se manifiesta la IC, algunas investigaciones sugieren que ir al colegio es una actividad particularmente estresante para los niños inhibidos (Coplan y Arbeau, 2008; Evans, 2001), lo que hace que se impliquen menos en actividades sociales (Kochanska, 1998) y puedan experimentar dificultades de adaptación a largo plazo. De hecho, los niños inhibidos son evaluados por sus maestros con mayor número de problemas emocionales que sus compañeros (Bohlin, Hagekull y Andersson, 2005; Coplan, Arbeau y Armer, 2008). También, muestran habitualmente un comportamiento reservado e índices mayores de ansiedad durante el juego libre con sus compañeros de colegio (Coplan y Arbeau, 2008; Coplan, Prakash, O’Neil y Armer, 2004). Fuera del contexto escolar, las madres describen a sus hijos inhibidos como menos activos socialmente con sus semejantes y más propensos a jugar solos en casa con un amigo (Coplan, DeBow, Schneider y Graham, 2009), lo que aumenta el riesgo de presentar malas relaciones, rechazo y exclusión de sus iguales (Coplan et al., 2004). De hecho, uno de los criterios que definen a los niños con alta IC es la presencia de bajas tasas de interacción con sus iguales (Monjas y Caballo, 2002). En cuanto a la prevalencia de la IC, los datos registran que aproximadamente el 15\% de los niños son extremadamente inhibidos y responden con miedo y conductas de retirada ante lugares, personas y objetos desconocidos (Kagan, 1997). Del mismo modo, los estudios revelan que cerca de un $10 \%$ de los niños que en la edad preescolar presentan IC continúan expresándola durante la infancia, adolescencia y adultez (Kagan y Snidman, 2004).

Uno de los aspectos más importantes en la IC es la inhibición del habla, ya que supone un factor esencial para el estudio de la respuesta de inhibición ante la novedad y uno de los índices observables más utilizados para su evaluación (Kagan, 1994). Las investigaciones muestran que los niños 
inhibidos hablan menos, utilizan menos palabras, realizan intervenciones más breves y abarcan menos temas durante una conversación. Además, los niños con IC responden a preguntas de los maestros utilizando silencios o monosílabos más que los niños clasificados como desinhibidos (Evans, 1987; Kagan, Reznick, Snidman, Gibbons et al., 1988). Como resultado, cuando los maestros quieren que hablen deben insistirles más que a los demás niños (Evans, 1987). En relación con la inhibición del habla, Evans (1993) y London y Sommers (1979), señalan que además de hablar menos, los niños inhibidos presentan un lenguaje cualitativamente diferente, obteniendo puntuaciones más bajas en diferentes medidas de articulación, automaticidad, comprensión y producción de sintaxis, morfología y semántica.

\section{Relación de la IC con los trastornos de ansiedad}

La relación entre IC y los trastornos psicopatológicos aparece en numerosas investigaciones (Van Brakel y Muris, 2006). En esta línea, los estudios relacionan la IC con los trastornos de ansiedad (Chronis-Tuscano et al., 2009; Essex et al., 2010; Hirshfeld-Becker et al., 2007; Hudson y Dodd, 2012; Hudson, Dodd, Lyneham y Bovopoulous, 2011; Orgilés, Méndez, Espada, Carballo y Piqueras, 2012; Sportel, 2013.). De modo que, cuando se trata de niños que permanecen inhibidos de forma estable a lo largo del tiempo (Hirshfeld-Becker et al., 1992), la IC se contempla como un marcador conductual de vulnerabilidad biológica para el desarrollo de trastornos de ansiedad (Biederman, HirshfeldBecker, Rosenbaum, Christine et al., 2001; Kagan, Reznick y Snidman, 1988). Asimismo, aunque existen estudios que evidencian que las dificultades derivadas del temperamento inhibido predisponen también a la aparición de síntomas depresivos, estos señalan que dicha relación está mediada por la ansiedad (Muris, Merckelbach, Schmidt, Gadet y Bogie, 2001; Muris, van Brakel, Arntz y Schouten, 2011; Sportel, 2013).

La edad normal de inicio en la infancia de un trastorno de ansiedad se sitúa entre los 7 y 12 años (Last, Perrin, Hersen y Kazdin, 1992). Sin embargo, la primera adquisición de los temores puede ser mucho más temprana (Ohman, 1985). En este sentido, Hudson et al. (2011) señalan que los niños inhibidos de 4 años son significativamente más propensos a ser diagnosticados, a los 6 años, con fobia social y trastorno de ansiedad generalizada frente a los no inhibidos. Además, un estudio realizado en España con una muestra de niños y adolescentes de entre 8 y 14 años mediante autoinformes, muestra cómo los trastornos de ansiedad evolucionan con la edad. De manera que los niños mayores obtienen menos síntomas de ansiedad por separación, de trastorno obsesivocompulsivo y de pánico, pero mayores manifestaciones de fobia social y de ansiedad generalizada (Orgilés et al., 2012). Respecto a las diferencias según el sexo, Orgilés et al. (2012) afirman que las niñas manifiestan más ansiedad que los niños en todos los trastornos de ansiedad, excepto en el trastorno obsesivo-compulsivo.

Aunque la prevalencia de los trastornos de ansiedad cambia considerablemente en función de la edad, de los instrumentos de evaluación o de la fuente de información, se estima que entre un 15 y un $20 \%$ de niños y adolescentes desarrollan un trastorno de ansiedad; siendo la ansiedad por separación, junto a las fobias específicas, los más frecuentes en población infanto-juvenil (Beesdo, Knappe y Pine, 2009). No obstante, aunque algunos estudios encuentran una prevalencia menor (Costello, Mustillo, Erkanli, Keeler y Angold, 2003; Meltzer, Gatward, Goodman y Ford, 2000), es necesario señalar que otros trabajos epidemiológicos indican que los trastornos de ansiedad son los problemas psicológicos más diagnosticados en niños y adolescentes españoles y los de mayor demanda asistencial (Echeburúa y Corral, 2009).

\section{Relación de la IC y los trastornos de ansiedad con otras variables}

Los estudios citados relacionan la IC con la etiología de los trastornos de ansiedad en la infancia. Pero ya que los datos demuestran que no todos los niños ansiosos presentan un comportamiento inhibido y no todos los niños con IC sufren patologías relacionadas con la ansiedad, resulta necesario conocer qué otros factores pueden estar implicados en el origen, desarrollo y mantenimiento de la relación entre IC y los trastornos de ansiedad. Ante esto, cabe la pregunta de en qué condiciones los niños con IC desarrollan trastornos de ansiedad. Para dar respuesta a esta cuestión, los estudios analizan la existencia de otras variables que pueden ser mediadoras de esta relación.

En esta línea, las investigaciones señalan que los niños de padres con algún trastorno de ansiedad presentan un mayor riesgo de manifestar IC y desarrollar un trastorno de ansiedad en edades tempranas (Hirshfeld-Becker, Micco, Henin, Bloomfield, Biederman y Rosenbaum, 2008; Hudson et al., 2011; Muris et al., 2011). De tal manera que según Biederman et al. (1993), en los menores inhibidos existe un mayor riesgo de padecer trastornos de ansiedad en ellos y en sus familias.

Del mismo modo, el estilo educativo de los padres es otra variable de riesgo implicada en el desarrollo de los 
trastornos de ansiedad (Rapee, 2001). Rapee señala que cuando los padres se muestran poco afectivos en la relación con sus hijos, les exteriorizan poca aceptación y un bajo nivel de afinidad, generan una mayor probabilidad de desarrollar una relación insegura, que a su vez posibilita el desarrollo de trastornos de ansiedad. En este mismo sentido, postula que una protección excesiva y un alto control de los padres pueden transmitir al niño un mensaje de que el mundo es un lugar peligroso y reducir el contacto del niño con estímulos novedosos o eventos amenazantes, minimizando las experiencias del niño y pudiendo reforzar la dependencia hacia los demás. Otros estudios apoyan este extremo (McLeod, Wood y Weisz, 2006; Wood, McLeod, Sigman, Hwang y Chu, 2003), sin embargo pocos arrojan luz sobre la naturaleza de la dirección de esta asociación. Rubin, Cheah y Fox (2001) informan que un pequeño control parental durante una tarea estructurada se asocia con un aumento de conductas de IC en niños preescolares. Aunque estos estudios señalan la posibilidad de que las prácticas de crianza moderan la relación entre el temperamento y la vulnerabilidad psicológica posterior de los niños, resulta evidente, que se necesita más investigación para entender mejor la contribución de los factores específicos de crianza (Thirlwall y Creswell, 2010). Ya que el comportamiento de los padres, según los estudios realizados, podría entenderse como una causa de la ansiedad en los niños, como una respuesta de los padres a la ansiedad de sus hijos o como la expresión de la propiedad ansiedad de los padres (Fox, Henserson, Marshall, Nichols y Ghera, 2005).

Igualmente, los resultados revelan que hay una relación entre los trastornos de ansiedad de la madre y los altos niveles de crítica hacia sus hijos, una de las dimensiones del constructo de emoción expresada. A su vez, los altos niveles de crítica se relacionan con el aumento de la posibilidad de presentar IC y un mayor número de psicopatologías en niños de 4 a 10 años (Hirshfeld, Biederman, Bordy, Faraone y Rosenbaum, 1997). En esta línea, se hallan puntuaciones más altas de emoción expresada en los padres cuyos hijos presentan trastornos de ansiedad (García-López, Muela, Espinosa-Fernández y Díaz-Castela, 2009). Por último, la variable estrés maternal en la infancia, es otra variable que también podría estar asociada con los trastornos de ansiedad en la adolescencia (Essex et al., 2010).

Los estudios apuntan a que no sólo los trastornos de ansiedad de los padres, el estilo educativo, la emoción expresada o el estrés maternal son variables que puedan relacionarse con los trastornos de ansiedad. Si no que hay otros factores de vulnerabilidad como un apego inseguro entre padres e hijos, los estilos negativos de crianza, las experiencias de aprendizaje específicos o los sucesos vitales estresantes que pueden contribuir a ello (Muris et al., 2011; Rapee, Schniering y Hudson, 2009). Del mismo modo, otras muchas variables han sido estudiadas para comprobar su influencia en la IC y los trastornos de ansiedad, aunque no se han obtenido diferencias significativas en ellas. Entre algunas de estas variables, se encuentran la expresividad y cohesión familiar, el orden de nacimiento o el número de hermanos (Hirshfeld-Becker, Biederman, Faraone, Segool, Buchwald y Rosenbaum, 2004) o la dependencia al alcohol y las drogas de los padres (Biederman, Hirshfeld-Becker, Rosenbaum, Perenick, Wood y Faraone, 2001).

$\mathrm{Al}$ igual que existen variables que pueden favorecer la presencia de IC y el desarrollo de trastornos de ansiedad, hay un consenso en considerar otros factores que actúan de protección en el desarrollo de la ansiedad infantil, variables tanto de carácter externo, familiar y socioambiental, como interno, genético y cognitivo (Donovan y Spense, 2000; Vasey y Dadds, 2001). Se sabe que todos estos factores interactúan entre sí y, dependiendo de variables tales como las características del contexto, la vulnerabilidad individual, la fase de desarrollo, así como la presencia o ausencia de otros factores de riesgo o protección, pueden ser o no el resultado del desarrollo de problemas de inhibición y ansiedad en el niño (Espinosa-Fernández, 2009). De todas formas, algunos autores consideran que aún son necesarios nuevos estudios en los que se examine los efectos aditivos e interactivos de la IC junto a una amplia gama de otros factores de vulnerabilidad en el desarrollo de la ansiedad patológica de los jóvenes (Hirshfeld-Becker, Micco, Henin et al., 2008; Hirshfeld-Becker, Micco, Simoes y Henin, 2008).

\section{Procedimientos de evaluación de la IC}

Debido a que la IC es una variable cuya presencia ocasiona malestar emocional en los niños, afectando a su competencia social y predisponiéndolos a padecer trastornos de ansiedad a ellos y a sus familias (Biederman et al., 1993); su evaluación y detección precoz podría proporcionar un medio clínicamente útil para concretar qué niños son más propensos a padecer trastornos de ansiedad. Por ello, el estudio y evaluación de la IC parece una estrategia adecuada para hallar las características adicionales que puedan predecir un riesgo alto de desarrollar trastornos de ansiedad (Biederman, Hirshfeld-Becker, Rosenbaum, Christine et al., 2001) y así prevenir su aparición temprana (Morizot y Vitaro, 2003) o poder desarrollar estrategias de intervención y programas educativos eficaces (Taboada, Ezpeleta y de la Osa, 1998) de los que puedan beneficiarse los programas dirigidos a la 
prevención de los trastornos de ansiedad (Mateu-Martínez, Piqueras, Jiménez-Albiar, Espada, Carballo y Orgilés, 2013; Rosenbaum et al., 2000). En esta línea, Thirlwall y Creswell (2010) señalan que a pesar de estar bien documentada en la bibliografía las consecuencias negativas que tiene la presencia de un trastorno de ansiedad en la vida de los niños, las estrategias de prevención aún deben de mejorar en cuanto a su eficacia (Klaus Minde, 2013; Rapee, Kennedy, Ingram, Edwards y Sweeney, 2005). Para ello, las teorías y modelos contemporáneos sobre la etiología y desarrollo de los trastornos de ansiedad deben ser completados con la aportación de nuevos datos.

Dadas las ventajas que una detección precoz de la IC podría tener en los niños y teniendo en cuenta que ésta es sensible a la influencia de variables contextuales (Goldsmith et al., 1987), la etapa preescolar parece un momento idóneo para llevar a cabo su evaluación. Ya que es una etapa de la infancia lo suficientemente adelantada como para que la detección pueda ser considerada temprana y, al mismo tiempo, es lo suficientemente tarde como para poder obtener información de diferentes contextos, incluido el colegio.

Para la evaluación de la IC suele utilizarse tres procedimientos: las pruebas de laboratorio, la observación directa y los instrumentos de lápiz y papel (Ballespí y Jané, 2002). En las pruebas de laboratorio, los niños son expuestos a estímulos novedosos y se miden tanto comportamientos externos como variables fisiológicas; y aunque es un método valioso, necesita mucho tiempo para identificar a los jóvenes en riesgo (Van Brakel y Muris, 2006). En la observación de entornos naturales, Ballespí y Jané (2002) ponen de manifiesto la necesidad de utilizar instrumentos de registro para dejar constancia de lo observado en los diferentes contextos, casa o colegio, aspecto que puede llegar a ser bastante costoso. Por último, en los instrumentos de lápiz y papel, la obtención de información de padres y de maestros presenta notables ventajas en comparación con los registros psicofisiológicos y la observación directa, ya que muestran una elevada economía en el procedimiento, un alto número de información recogida y los datos tienen una alta validez ecológica. Hirshfeld-Becker, Micco, Simoes et al. (2008) manifiestan que estos instrumentos pueden contener sesgos retrospectivos o deseabilidad social, por lo que señalan la conveniencia de utilizarlos junto a los procedimientos de observación directa.

Un análisis más profundo, de las pruebas de lápiz y papel, indica que la utilización de autoinformes no resulta adecuada para la evaluación de la IC en preescolares, ya que estos niños aún no son capaces de informar sobre ciertas variables personales. Por tanto, para la evaluación en estas edades se utilizan cuestionarios y escalas basados en la información que suministran padres y maestros. En esta línea, una revisión de los instrumentos pone de manifiesto la escasez de pruebas específicas de evaluación de la IC en niños tan pequeños. Ballespí (2004) señala el uso de las siguientes pruebas en edades tempranas: la Escala de Inhibición para Padres de Asendorpf (1990), Instrumento de Inhibición Conductual de Muris, Merckelbach, Wessel y van de Ven (1999), la Escala de Bohlin, Bengtsgard y Andersson (2000), Cuestionario de Inhibición Conductual de Bishop, Spence y McDonald (2003) y la Escala de Inhibición Conductual para Preescolares de Ballespí, Jané, Riba y Doménech-Llaberia (2003). Siendo esta última, según Ballespí (2004), la única disponible en español y adaptada a la población española, que abarca las edades preescolares de 3 a 6 años y cuyos ítems son los más adecuados para el conjunto de signos que pueden observarse en entornos naturales.

Igualmente, hay que hacer mención a algunas otras pruebas entre los instrumentos de lápiz y papel, como el Inventario del Comportamiento de Niños para Padres y para Maestros-Cuidadores de Achenbach y Rescorla (2000). Aunque este inventario no incluye subescalas específicas de IC y no ha sido utilizado en trabajos empíricos para evaluar estos rasgos, sin embargo, algunos de sus ítems hacen clara referencia a la IC o aspectos relacionados con este fenómeno (Ballespí, 2004), pues mide numerosas variables adaptativas e inadaptativas en los niños que pueden estar relacionadas con la presencia o ausencia de IC. Del mismo modo, existen otros instrumentos, como las Escalas de Áreas de Conductas-Problema de García Pérez y Magaz (2000) y el Sistema de Evaluación de la Conducta de Niños y Adolescente de Reynolds y Kamphaus (2004), que evalúan una amplia gama de dimensiones patológicas (problemas de conducta, trastornos emocionales, problemas de personalidad) y dimensiones adaptativas (habilidades sociales, adaptabilidad) que podrían estar también relacionadas con la IC. Además, estos dos instrumentos están disponibles tanto para padres como para maestros, están adaptados a la población española y a las edades preescolares y su utilización podría aumentar la variabilidad de factores evaluados en relación a la IC y posibilitar, de este modo, la comparación con los datos obtenidos en los instrumentos de evaluación anteriormente citados.

Por tanto, la evaluación temprana y exhaustiva de la IC, como una de las variables implicadas en el desarrollo de la ansiedad, se convierte un interesante recurso de prevención primaria (Mick y Telch, 1998). Ya que, como se ha señalado, los trastornos de ansiedad en la infancia no suelen remitir sin tratamiento (Costello et al., 2003), pueden continuar en la edad adulta (Kagan et al., 2007), se relacionan con la 
presencia de dificultades psicosociales en el niño (Brent et al., 1998; Messer y Beidel, 1994) y aumentan el riesgo de desarrollar otros trastornos psiquiátricos comórbidos (Curry y Murphy, 1995; Thapar y McGuffin, 1997).

\section{Discusión}

La revisión bibliográfica realizada muestra que la investigación científica sobre la IC ha sido un campo de estudio prolífico durante las últimas décadas. No obstante, todavía necesita responder a numerosos interrogantes sobre las características propias de la IC y de su relación con los trastornos de ansiedad. En este sentido, los estudios deberían explicar porqué no todos los niños ansiosos muestran un comportamiento inhibido ni todos los niños con IC sufren trastornos de ansiedad.

Para ello, los estudios tendrían que seguir profundizando en las variables ya estudiadas sobre el origen, desarrollo y mantenimiento de los factores relativos a la IC y los vinculados con los trastornos de ansiedad. En esta línea, se ahondaría en el conocimiento tanto de las variables propias del niño (v.g. rasgos de personalidad, ansiedad, edad, sexo o habilidades verbales) como en la influencia de los factores ambientales (v.g. contextos, trastornos de ansiedad en los padres, emoción expresada, apego inseguro, estilos de crianza negativos, experiencias de aprendizaje específicos, sucesos vitales estresantes o ansiedad maternal). Al mismo tiempo, sería necesario realizar investigaciones que incluyeran nuevas variables de estudio de carácter intelectual, madurativo, conductual y socioemocional que pudieran estar asociadas con la IC y los trastornos de ansiedad (v.g. quejas somáticas, adaptabilidad al medio, agresividad, hiperactividad, atención). El objetivo final de este proceso, sería conocer de manera exhaustiva la relación de la IC con los trastornos de ansiedad. Por lo que al estudiar las variables, sería prioritario examinar la direccionalidad de sus influencias y los efectos conjuntos de sus posibles interacciones (Hirshfeld-Becker, Micco, Henin et al., 2008; Hirshfeld-Becker, Micco, Simoes et al., 2008). De tal forma, que se pudieran descubrir las combinaciones de factores que protegen y perjudican conjuntamente esta relación, en línea con las aportaciones de Espinosa-Fernández (2009), Donovan y Spense (2000) y Vasey y Dadds (2001).

Para llevarlo a la práctica, y debido al carácter estable que la IC presenta durante la infancia, adolescencia y adultez (Kagan y Snidman, 2004), sería necesario realizar estudios longitudinales sobre la IC, de su relación con los trastornos de ansiedad y cómo éstos cambian con la edad (Orgilés et al.,
2012), abarcando el mayor rango posible de tiempo. Además, en dichos estudios, se debería aumentar la variabilidad de los informadores incluyendo también a los padres, ya que generalmente los datos recogidos en el ambiente familiar son facilitados principalmente por las madres. Este hecho posibilitaría el análisis de las semejanzas y diferencias entre los progenitores y las influencias individuales y conjuntas que generaran.

En cuanto a los procedimientos de evaluación, resultaría imprescindible que las futuras investigaciones trabajaran en la creación o perfeccionamiento de nuevos y sencillos instrumentos para la evaluación precoz de la IC. De esta manera, se podría trabajar en la identificación temprana de los trastornos de ansiedad en niños, convirtiendo este posible adelanto en un interesante recurso para prevenir su aparición (Morizot y Vitaro, 2003). Además, sería necesario que este nuevo conocimiento teórico se utilizara para desarrollar o perfeccionar programas de intervención escolar sobre la IC y los trastornos de ansiedad (Taboada et al., 1998) y así poder mejorar su eficacia (Rapee et al., 2005) en la infancia. En este sentido, las investigaciones señalan que las intervenciones en la etapa preescolar tienen un alto impacto en los niños, ya que a estas edades son muy susceptibles a las influencias ambientales (Urzúa, Ramos, Alday y Alquinta, 2010).

En definitiva, para conocer y evitar las influencias negativas de la IC y los trastornos de ansiedad en los niños, las futuras investigaciones deberían realizar estudios longitudinales, que incluyeran el mayor número posible de fuentes de información y que utilizasen instrumentos de evaluación de la IC en edades cada vez más precoces; lo que posibilitaría anticipar el conocimiento de un mayor riesgo de desarrollar trastornos de ansiedad en la infancia. Además, estos estudios deberían profundizar en variables ya estudiadas e investigar otras nuevas para descubrir la direccionalidad de las influencias y los efectos aditivos de las interacciones entre variables. Por último, las investigaciones deberían aplicar el nuevo conocimiento en la creación y perfeccionamiento de programas de prevención de la IC y a la intervención temprana sobre los trastornos de ansiedad.

\section{Referencias}

Achenbach, T. M., y Rescorla, L. A. (2000). Manual for the ASEBA preschool forms and profiles. Burlington, VT: University of Vermont, Research Center for Children, Youth and Families.

Asendorpf, J. B. (1990). Development of inhibition during childhood: Evidence for situational specificity and a two factor model. Developmental Psychology, 26, 721-730. 
Ballespí, S. (2004). PAIC 3-6. Protocol d'avaluació de la inhibició conductual per nens de 3 a 6 anys. (Tesis doctoral, Universidad Autónoma de Barcelona). Recuperado desde http://ddd.uab.cat/pub/tesis/2005/ tdx-0620105-124401/sbs1de1.pdf

Ballespí, S., y Jané, M. C. (2002). ¿Cómo evaluar la inhibición conductual? Una revisión de instrumentos. Revista de Psiquiatría Infanto-juvenil, 1, 69-81. Clínica (pp. 41-78). Barcelona: Universidad Autónoma de Barcelona.

Ballespí, S., Jané, M. C., Riba, M. D., y Doménech-Llaberia, E. (2003). Escala de Inhibición conductual para preescolares, versión maestros (EICP-M): propiedades psicométricas. Psicothema, 15, 205-210.

Beesdo, K., Knappe, S., y Pine, D. S. (2009). Anxiety and anxiety disorders in children and adolescents: developmental issues and implications for DSM-V. Psychiatric Clinics of North America, 32, 483-524.

Biederman, J., Hirshfeld-Becker, D. R., Rosenbaum, J. F., Christine, H., Friedman, D., Snidman, N., Kagan, J., y Faraone, S. V. (2001). Further evidence of association between behavioral inhibition and social anxiety in children. American Journal of Psychiatry, 158, 1673-1679.

Biederman, J., Hirshfeld-Becker, D. R., Rosenbaum, J. F., Perenick, S., Wood, J., y Faraone, S. V. (2001). Lack of association between parental alcohol or drug addiction and behavioral inhibition in children. The American Journal of Psychiatry, 158, 1731-1733.

Biederman, J., Rosenbaum, J. F., Bolduc, E. A., Faraone, S. V., Chaloff, J., Hirshfeld, D. R., y Kagan, J. (1993). A 3-Year follow-up of children with and without behavioral inhibition. Journal of the American Academy of Child and Adolescent Psychiatry, 32, 814-821.

Bishop, G., Spence, S. H., y McDonald, C. (2003). Can parents and teachers provide a reliable and valid report of behavioral inhibition? Child Development, 74, 1899-1917.

Bohlin G., Bengtsgard K., y Andersson K. (2000). Social inhibition and overfriendliness as related to socioemotional functioning in 7-and 8-year-old children. Journal of Clinical Child Psychology, 29, 414-423.

Bohlin, G., Hagekull, B., y Andersson, K. (2005). Behavioral inhibition as a precursor of peer social competence in early school age: The interplay with attachment and non parental care. Merrill-Palmer Quarterly, 51, 1-19.

Brent, D., Kolko, D. J., Birmaher, B., Baugher, M., Bridge, J., Roth, C., y Holder, D. (1998). Predictors of treatment efficacy in a clinical trial of three psychosocial treatments for adolescent depression. Journal of the American Academy of Child and Adolescent Psychiatry, 37, 906-914.

Chronis-Tuscano, A., Degnan, K. A., Pine, D. S., Pérez-Edgar, K., Henderson, H. A., Díaz, Y., Raggi, V. L., y Fox, N. A. (2009). Stable early maternal report of behavioral inhibition predicts lifetime social anxiety disorder in adolescence. Journal of the American Academy of Child and Adolescent Psychiatry, 48, 928-935.

Coplan, R. J., y Arbeau, K. A. (2008). The stresses of a brave new world: Shyness and adjustment in kindergarten. Journal of Research in Childhood Education, 22, 377-389.

Coplan, R. J., Arbeau, K. A., y Armer, M. (2008). Don't fret, be supportive! Maternal characteristics linking child shyness to psychosocial and school adjustment in kindergarten. Journal of Abnormal Child Psychology, 36, 359-371.

Coplan, R. J., DeBow, A., Schneider, B. H., y Graham, A. A. (2009). The social behaviors of inhibited children in and out of preschool. British Journal of Developmental Psychology, 27, 891-905.

Coplan, R. J., Prakash, K., O’Neil, K., y Armer, M. (2004). Do you want to play? Distinguishing between conflicted-shyness and social disinterest in early childhood. Developmental Psychology, 40, 244 -258.

Costello, J., Mustillo, S., Erkanli, A., Keeler, G., y Angold A. (2003). Prevalence and development of psychiatric disorder in childhood and adolescence. Archive General of Psychiatry, 60, 837-844.

Craske, M. G. (1997). Fear and anxiety in children and adolescents. Bulletin of the Menninger Clinic, 61, 4-36.

Curry, J. F., y Murphy, L. B. (1995). Comorbidity of anxiety disorders. In J. S. March (Ed.), Anxiety disorders in children and adolescents (pp. 301-317). New York: Guilford Press.

Donovan, C. L., y Spence, S. H. (2000). Prevention of childhood anxiety disorders. Clinical Psychology Review, 65, 627-635.
Echeburúa, E., y Corral, P. (2009). Trastornos de ansiedad en la infancia $y$ adolescencia. Madrid: Pirámide.

Espinosa-Fernández, L. (2009). Ansiedad infantil e implicación de los padres: una revisión. Psicología Conductual, 17, 67-87.

Essex, M. J., Klein, M. H., Slattery, M. J., Goldsmith, H. H., y Kalin, N. H. (2010). Early risk factors and developmental pathways to chronic high inhibition and social anxiety disorder in adolescence. American Journal of Psychiatry, 167, 40-46.

Evans, M. A. (1987). Discourse characteristics of reticent children. Applied Psycholinguistics, 8, 171-184.

Evans, M. A. (1993). Communicative competence as a dimension of shyness. En K. H. Rubin y J. B. Asendorpf (Eds.), Social withdrawal, inhibition, and shyness in childhood (pp. 189-212). Hillsdale, NJ: Erlbaum.

Evans, M. A. (2001). Shyness in the classroom and home. En W. R. Crozier y L. E. Alden (Eds.), International handbook of social anxiety: Concepts, research and interventions relating to the self and shyness, (pp. 159-183). Westport, CT: Wiley.

Fox, N. A., Henserson, H. A., Marshall, P. J., Nichols, K. E., y Ghera, M. M. (2005). Behavioral inhibition: linking biology and behavior within a developmental framework. Annual Review of Psychology, 6, 235-262.

García-Coll, C., Kagan, J., y Reznick, J. S. (1984). Behavioral inhibition in young children. Child Development, 55, 1005-1019.

García-López, L. J., Muela, J. M., Espinosa-Fernández, L., y DíazCastela, M. M. (2009). Exploring the relevance of expressed emotion to the treatment of social anxiety disorder in adolescence. Journal of Adolescence, 32, 1371-1376.

García Pérez, E. M., y Magaz, A. (2000). EACP. Escalas Magallanes de Áreas de Conductas Problema. Bilbao: COHS, Consultores en Ciencias Humanas.

Goldsmith, H. H., Buss, A. H., Plomin, R., Rothbart, M. K., Thomas, A., Chess, S., Hinde, R. A., y McCall, R.B. (1987). Roundtable: What is temperament? Four approaches. Child Development, 58, 505-529.

Guerra, P., Martín, M. E., y Arnaiz, A. (2011). La inhibición conductual: El estado de la investigación. Revista de Psicología Universidad Viña del Mar, 1, 88-109.

Hirshfeld, D. R., Biederman, J., Bordy, L., Faraone, S. V., y Rosenbaum, F. (1997). Expressed emotion toward children with behavioral inhibition: associations with maternal anxiety disorder. Journal Academy Child Adolescent Psychiatry, 36, 910-917.

Hirshfeld-Becker, D., Biederman, J., Faraone, S. V., Segool, N., Buchwald, J., y Rosenbaum, J. (2004). Lack of association between behavioral inhibition and psycho-social adversity factors in children at risk for anxiety disorders. The American Journal of Psychiatry, 161, 547-555.

Hirshfeld-Becker, D. R., Biederman, J., Henin, A., Faraone, S. V., Davis, S., Harrington, K., y Rosenbaum, J. F. (2007). Behavioral inhibition in preschool children at risk is a specific predictor of middle childhood social anxiety: A five-year follow-up. Journal of Developmental and Behavioral Pediatrics, 2, 225-233.

Hirshfeld-Becker, D. R., Micco, J., Henin, A., Bloomfield, A., Biederman, J., y Rosenbaum, J. (2008), Behavioral inhibition. Depression and Anxiety, 25, 357-367.

Hirshfeld-Becker, D. R., Micco, J., Simoes, N. A., y Henin, A. (2008). High risk studies and developmental antecedents of anxiety disorders. American Journal of Medical Genetics, 148, 99-117.

Hirshfeld-Becker, D. R., Rosenbaum, J. F., Biederman, J., Bolduc, E. A., Faraone, S. V., Snidman, N., Reznick, J. S., y Kagan, J., (1992). Stable behavioral inhibition and its association with anxiety disorder. Journal of American Academy Child and Adolescence Psychiatry, 31, 103-111.

Hudson, J. L. y Dodd, H. F. (2012). Informing Early Intervention: Preschool Predictors of Anxiety Disorders in Middle Childhood. PLOS ONE, 7(8): e42359, 1-7. doi:10.1371/journal.pone.0042359

Hudson, J. L., Dodd, H. F., Lyneham, H. J., y Bovopoulous, N. (2011). Temperament and family environment in the development of anxiety disorder: Two-year follow-up. Journal of the American Academy of Child and Adolescent Psychiatry, 50, 1255-1264.

Kagan, J. (1994). Galen's prophecy: temperament in human nature. Oxford: Basic Books. 
Kagan, J. (1997). Temperament and the reactions to the un familiarity. Child Development, 68, 139-143.

Kagan, J., Reznick, S. J., y Snidman, N. (1987). The physiology and psychology of behavioral inhibition in children. Child Development, $58,1.459-1.473$.

Kagan, J., Reznick, S. J., y Snidman, N. (1988). Biological bases of childhood shyness. Science, 240, 167-171.

Kagan, J., Reznick, J. S., Snidman, N., Gibbons, J., y Johnson, M. O. (1988). Childhood derivates of inhibition and lack of inhibition to the unfamiliar. Child Development, 59, 1580-1589.

Kagan, J., y Snidman, N. (2004). The long shadow of temperament. Cambridge: MA Harvard University Press.

Kagan, J., Snidman, N., y Arcus, D. (1998). Childhood derivatives of high and low reactivity in infancy. Child Development, 69, 1483-1493.

Kagan, J., Snidman, N., Kahn, V., y Towsley, S. (2007). The preservation of two infant temperaments into adolescence. Monographs of the Society for Research in Child Development, 72, Serial n ${ }^{\circ} .287,1-75$.

Kerr, M., Lambert, W. W., Stattin, H., y Klackenberg-Larsson, I. (1994). Stability of inhibition in a Swedish longitudinal sample. Child Development, 65, 138-146.

Klaus Minde, M. D. (2013). Diagnosis and Cognitive Behavioral Treatment of Anxiety Disorders in Young Children. En E. A. Storch y D. McKay (Eds.), Handbook of Treating Variants and Complications in Anxiety Disorders (pp. 109-123). New York: Springer-Verlag.

Kochanska, G. (1998). Mother-child relationship, child fearfulness, and emerging attachment: A short-term longitudinal study. Developmental Psychology, 34, 480-490.

Last, C., Perrin. S., Hersen, M., y Kazdin, A. (1992). DSM-III-R anxiety disorders in children: Sociodemographic and clinical characteristics, Journal of the American Academy of Child and Adolescent Psychiatry, 31, 1070-1076.

London, S. J., y Sommers, R. K. (1979). Talkativeness and children's linguistic abilities. Language and Speech, 22, 269-275.

Mateu-Martínez, O., Piqueras, J. A., Jiménez-Albiar, M. I., Espada, J. P., Carballo, J. L., y Orgilés, M. (2013). Eficacia de un programa de prevención cognitivo-conductual breve del rechazo social en niños. Terapia psicológica, 31, 87-195.

Matthews, G., Deary, I. J., y Whiteman, M. C. (2003). Personality traits $\left(2^{a}\right.$ ed.). Cambridge, Reino Unido: Cambridge University Press.

McCleod, B. D., Wood, J. J., y Weisz, J. R. (2006). Examining the association between parenting and child anxiety: A meta-analysis. Clinical Psychology Review, 27, 155-172.

Meltzer, H., Gatward, R., Goodman, R., y Ford, T. (2000). The mental health of children and adolescents in Great Britain. London: The Stationary Office.

Messer, S. C., y Beidel, D. C. (1994). Psychosocial correlates of childhood anxiety disorders. Journal of the American Academy of Child and Adolescent Psychiatry, 33, 75-98.

Mick, M. A., y Telch, M. J. (1998). Social anxiety and history of behavioral inhibition in young adults. Journal of Anxiety Disorders, 12, 1-20.

Monjas, I., y Caballo, V. E. (2002). Psicopatología y tratamiento de la timidez en la infancia. En V. E. Caballo y M. A. Simón (Eds.), Manual de Psicología Clínica Infantil y del Adolescente (pp. 271-296). Madrid: Pirámide.

Morizot, J., y Vitaro, F. (2003). Temperament et components perturbateurs chez l'enfant: une reuve critique des etudes longitudinales. Bulletin de Psychiatry, 56, 69-78.

Muris, P., Merckelbach, H., Schmidt, H., Gadet, B., y Bogie, N. (2001). Anxiety and depression as correlates of self-reported behavioral inhibition in normal adolescents. Behaviour Research and Therapy, 39, 1.051-1.061.

Muris, P., Merckelbach, H., Wessel, I., y van de Ven, M. (1999). Psychopathological correlates of self-reported behavioural inhibition in normal children. Behaviour Research and Therapy, 37, 575-584.

Muris, P., van Brakel, A., Arntz, A., y Schouten, E. (2011). Behavioral inhibition as a risk factor for the development of childhood anxiety disorders: a longitudinal study. Journal of Child and Family Studies, $20,157-170$.

Ohman, A. (1985). Face the beast and fear the face: Animal and social fears as prototypes for evolutionary analysis of emotion, Psychophysiology, 23, 123-145.

Orgilés, M., Méndez, X., Espada, J. P., Carballo, J. L., y Piqueras, J. A (2012). Síntomas de trastornos de ansiedad en niños y adolescentes: Diferencias en función de la edad y el sexo en una muestra comunitaria. Revista de Psiquiatría y Salud Mental, 5, 115-120.

Pfeifer, M., Goldsmith, H. H., Davidson, R. J., y Rickman, M. (2002). Continuity and change in inhibited and uninhibited children. Child Development, 73, 1474-1485.

Rapee, R. (2001). The development of generalised anxiety. En M. Vaey y M. Dadds (Eds.), The developmental psychopathology of anxiety (pp. 481-503). Nueva York: Oxford University Press.

Rapee, R. M., Kennedy, S., Ingram, M., Edwards, S., y Sweeney, L. (2005). Prevention and early intervention of anxiety disorders in inhibited preschool children. Journal of Consulting and Clinical Psychology, 7, 488-497.

Rapee, R. M., Schniering, C. A., y Hudson, J. L. (2009). Anxiety disorders during childhood and adolescence. Annual Review of Clinical Psychology, 5, 311-341.

Reynolds, C. R., y Kamphaus, R. W. (2004). Behavior assessment system for children ( $2^{\mathrm{a}}$ ed.). Circle Pines, MN: American Guidance Service.

Rosenbaum, J. F., Biederman, J., Hirshfeld-Becker, D. R., Kagan, J., Snidman, N., Friedman, D., Nineberg, A., Gallery, D. J., y Faraone, S. V. (2000). A controlled study of behavioral inhibition in children of parents with panic disorder and depression. American Journal Psychiatry, 157, 2002-2010.

Rubin, K. H., Cheah, C. S. L., y Fox, N. (2001). Emotion regulation, parenting, and display of social reticence in preschoolers. Early Education and Development, 12, 97-115.

Spitz, R. (1961). El primer año de la vida del niño. Madrid: Aguilar.

Sportel, B. E. (2013). Adolescents at risk for social and test anxiety: who are at risk and how can we help? [Versión de University of Groningen Library]. Recuperado desde http://irs.ub.rug.nl/ppn/357239237

Taboada, A. M., Ezpeleta, L., y de la Osa, N. (1998). Trastornos por ansiedad en la infancia y adolescencia: factores de riesgo. Ansiedad y estrés, 4, 1-16.

Thapar, A., y McGuffin, P. (1997). Anxiety and depressive symptoms in childhood: A genetic study of comorbidity. Journal of Child Psychology and Psychiatry, 38, 651-656.

Thirlwall, K., y Creswell, C. (2010). The impact of maternal control on children's anxious cognitions, behaviors and affect: An experimental study. Behaviour Research and Therapy, 48, 1041-1046.

Urzúa, A., Ramos, M., Alday, C., y Alquinta, A. (2010). Madurez neuropsicológica en preescolares: Propiedades psicométricas del test CUMANIN. Terapia Psicológica, 28, 13-25.

Van Brakel, A., y Muris, P. (2006). A brief scale for measuring "behavioral inhibition to the unfamiliar" in children. Journal of Psychopathology and Behavioral Assessment, 28, 79-84.

Vasey, M., y Dadds, M. R. (2001). An introduction to the developmental psychopatology of anxiety. En M. W. Vasey y M. R. Dadds (Dirs.), The developmental psychopathology of anxiety (pp. 3-26). Oxford University Press.

Vreeke, L. J., y Muris, P. (2012). Relations between behavioral inhibition, big five personality factors, and anxiety disorder symptoms in nonclinical and clinically anxious children. Child Psychiatry and Human Development, 43, 884-894.

Wood, J., McLeod, B. D., Sigman, M., Hwang, W. C., y Chu, B. C. (2003). Parenting y childhood anxiety: Theory, empirical findings and future directions. Journal of Child Psychology and Psychiatry and Allied Disciplines, 44, 134-151. 